von Photokopien dient, sollen hier noch nähere Angaben gemacht werden.

Das Copyrapid-Negativpapier enthält einerseits auf gleiche Flächen berechnet etwa 1000-mal mehr Silber (als Halogensilber vorhanden) als das CopyrapidPositivpapier (als Silberkeime). Andererseits ist - wie durch vergleichende praktische Versuche festgestellt wurde - das Keimsilber der Positivschicht 10-Millionen-mal aktiver als die entwickelten Silberkörner der Copyrapid-Negativschicht. Man kann näherungsweise annehmen, daß das Positiv- (gleich Übertrag-) Papier trotz seines geringen Silbergehaltes 10000-mal mehr Keime enthält als das Negativpapier und daß darauf die sehr rasche Silberausscheidung in dieser Schicht zurückzuführen ist. Andererseits kann man aber zeigen, daß auch die „Keime“ der Negativschicht die Reduktion der Silbersalze beschleunigen können. Ein unbelichtetes Copyrapid-Negativpapier, das man mit einem Natriumthiosulfat enthaltenden Entwickler behandelt, ohne es dabei mit dem Positivpapier in Kontakt zu bringen, verschleiert ziemlich stark. In diesem Falle wandern die sich bildenden löslichen Silbersalze nicht genügend rasch $a b$ und werden daher durch die

2 In den dem Copyrapid-Verfahren zugrunde liegenden Patenten wird erstmalig die Verwendung von Silberkeimen zur Beschleunigung der Silberabscheidung in der Positivschicht erwähnt. Siehe z. B. Schweiz. Patent 240472 , Norweg. Patent 66994 der ehemaligen IG. Farbenindustrie A.G. (Priorit. 24. 1. 1941).
Keime der Negativschicht, z. B. geringe Spuren Schleiersilber, beeinflußt.

Entwickelt man die Copyrapid-Negativschicht in Kontakt mit der Positivschicht, so tritt eine merkbare Reduktion der gelösten Silbersalze in der Negativschicht nur in den Mitteltönen ein. An diesen Stellen befinden sich in der Negativschicht neben verhältnismäßig vielen Silberkeimen (entwickeltes Bildsilber) gelöste Silbersalze in genügend hoher Konzentration. Praktisch bedeutet dies eine Gradationsveränderung des Copyrapid-Negativs gegenüber einem normal entwickelten und fixierten Bild des gleichen Papieres.

In den vorangehenden Ausführungen wurde angenommen, daß die Keime der Positivschicht aus Silber bestehen. Das ist aber nicht notwendig, denn als Keime wirken sehr viele Substanzen, wie verschiedene kolloide Metalle oder Metallsulfide. Man kann die Keime z. B. auch erst während des Prozesses entstehen lassen, indem man z. B. der Positivschicht einen Stoff zusetzt, der mit den eindiffundierenden Silbersalzen spurenweise Schwefelsilber liefert.

Für die Geschwindigkeit der Silberreduktion spielen auch noch andere Faktoren eine Rolle, z. B. die Art des Bindemittels der Schichten sowie bestimmte $\mathrm{Zu}$ sätze zur Emulsion usw. Für die praktische Verwertbarkeit der Silbersalzdiffusionsverfahren ist aber mit maßgebend die technische Möglichkeit, die Keime in den beiden Schichten hinsichtlich Menge und Anzahl günstig verteilen zu können.

\title{
Vertikaler Eberhard-Effekt und Schwarzschild-Effekt beim Agfacolorfilm
}

\author{
Von W. BEHRENDT \\ Aus dem wissenschaftlichen Laboratorium der Agfa-Photofabrik, Leverkusen-Bayerwerk \\ (Z. Naturforschg. 6 a, 382-384 [1951]; eingegangen am 4. April 1951) \\ Herrn Professor Dr. John Eggert zum 60. Geburtstage gewidmet

\begin{abstract}
Bei Mehrschichtenfarbfilmen wird bei verschieden starker Belichtung der einzelnen Schichten (stark gefärbtes Licht) die schwächer belichtete Schicht unterentwickelt. Dieser Effekt ist genau wie der normale Eberhard-Effekt (Nachbareffekt) zu erklären und wird daher als vertikaler Eberhard-Effekt bezeichnet.

Der Schwarzschild-Effekt wirkt sich in den einzelnen Schichten des Mehrschichtenfarbfilmes verschieden aus. Praktisch treten störende Farbverschiebungen bei langen Aufnahmebelichtungen mit Umkehrfilm und langen Kopierbelichtungen mit Positivfilm auf.
\end{abstract}

I $m$ folgenden soll über zwei Effekte berichtet werden, die aus der Literatur der Schwarzweiß-Photographie bekannt sind, aber im Mehrschichtenfilm in spezifischen Wirkungen bemerkbar werden. Dabei wollen wir uns auf solche Wirkungen beschränken, die praktisch eine gewisse Bedeutung haben und sich teils günstig bemerkbar machen, teils durch Gegenmaßnahmen bekämpft werden müssen.

Unter dem Namen Eberhard-Effekt (Nachbareffekt) versteht man die Tatsache, daß die entwickelbaren 
Schwärzungen benachbarter Stellen der Emulsionsschicht sich gegenseitig beeinflussen. Vor allem an den Berührungskanten von stark und schwach belichteten Filmstellen wird die schwächer belichtete Seite unterentwickelt. Man sieht also auf der hellen Seire der Kante einen Streifen, der noch heller als die weiter entfernten Stellen ist. Die Ursache liegt in der erhöhten Entwicklerausnutzung und starken Bromkalibildung an stark geschwärzten Filmstellen, die im Umkreis von einigen Zehntel Millimetern noch wirksam ist.

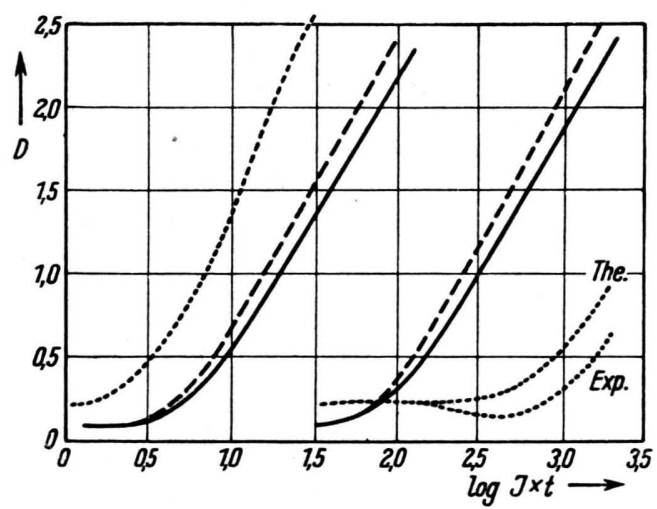

Abb. 1. Vertikaler Eberhard-Effekt bei Agfacolor-Positiv. Ordinate: Einzelschichtdichten.

$$
\begin{array}{ll}
\ldots \ldots \ldots \ldots \ldots & \text { Gelb. } \\
\ldots \ldots \ldots \ldots & \text { Purpur. } \\
& \\
&
\end{array}
$$

Links: Belichtung mit blauem Licht.

Rechts: Belichtung mit gelben Licht.

Auch im Mehrschichtenfilm kann man gelegentlich Effekte in dieser Form beobachten, besonders dann, wenn Film und Entwickler nicht bewegt werden. Interessanter als diese Beeinflussung innerhalb der Filmebene ist die Nachbarwirkung senkrecht dazu, also die Wirkung von einer Schicht auf die andere. Hier kann man einen Effekt dann beobachten, wenn eine Schicht wesentlich stärker als die andere belichtet ist, wenn also mit. stark gefärbtem Licht belichtet wurde. Einen solchen Fall zeigt Abb. 1.

Ein Agfacolor-Positivkeil wurde einmal mit bläulichem, das andermal mit stark gelb gefiltertem Licht aufgenommen. Die Messung geschah am Farbdichtemesser, dessen Meßwerte auf normierte (grauäqui-

1 Einzelheiten über die Messung bzw. Errechnung von Einzelschichtdichten siehe B e h rendt, Wissensch. Veröffentl. Agfa 7, 8 [1951]. Zur Definition von Einzelschichtdichten (analytic densities) siehe auch $\mathrm{Heymer}$ u. S u n d h o ff, Wiss. Veröff. Agfa 5, 62 [1937]; Komitee für Farbsensitometrie der SMPTE, J. Soc. Motion Picture Tel. Engr. 54, 653 [1950]. valente) Einzelschichtdichten umgerechnet wurden ${ }^{1}$. Bei der rechten Kurvenschar ist durch die Abschwächung des blauen Lichtes die Gelbkurve stark nach rechts verschoben. Theoretisch war nach der Dichte des benutzten Filters die mit The. bezeichnete Gelbkurve zu erwarten, während das Experiment die mit Exp. bezeichnete Kurve ergab, die unter der theoretischen Kurve liegt. Im ersten Teil der Kurve fällt diese sogar unter den Wert des Schleiers.

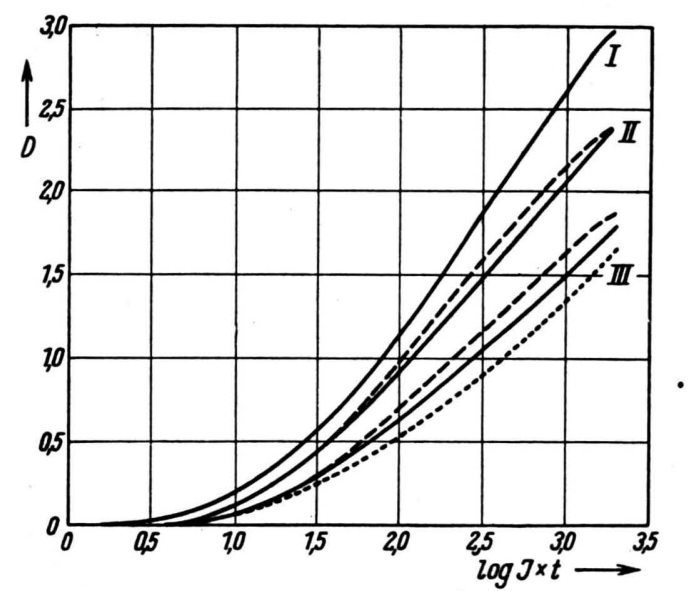

Abb. 2. Vertikaler Eberhard-Effekt bei Agfacolor-Negativ. Ordinate: Einzelschichtdichten.

I Blaugrünschicht allein (Einschichtfilm).

II Blaugrünschicht + Purpurschicht (Zweischichtenfilm). III Blaugrün- + Purpur- + Gelbschicht (Dreischichtenfilm mit Filtergelbschicht).

In Analogie zum normalen Eberhard-Effekt kann man diesen Effekt als vertikalen Eberhard-Effekt bezeichnen. Praktisch spielt er nur bei extrem gefärbten Filmstellen eine Rolle und erhöht dort den vertikalen Kontrast, also die Farbsättigung. Man kann diese Wirkung also im allgemeinen als günstig bezeichnen.

Auch bei gleichmäßiger Belichtung aller Schichten, also bei Belichtung mit „weißem“ Licht (d.h. für die betreffende Filmsorte weißes Licht) muß der Effekt auftreten; dort kann man ihn aber nur untersuchen, wenn man die Einzelschichten getrennt untersuchen kann, praktisch also nur in der Rohfilmfabrik. Ein solcher Fall ist in Abb. 2 dargestellt.

Hier wurde ein Film in den einzelnen Fabrikationsstadien sensitometrisch ausgewertet, also nach jeder Aufbringung einer neuen Schicht ein Sensitometerkeil gemessen. Da zwischen Purpur- und Gelbschicht noch die Filtergelbschicht liegt, deren Absorption die darunter liegenden Schichten beeinflußt, wurden die Sensitometerkeile I und II hinter einem genau äquivalen- 
ten Gelbfilter belichtet. Keil III wurde ohne Gelbfilter belichtet, da dieser Film ja selbst eine Filtergelbschicht enthielt.

Betrachtet man die drei Blaugrünkurven, so sieht man außer einer Empfindlichkeitsverminderung eine Verflachung mit zunehmender Zahl der Schichten. Die Empfindlichkeitsverminderung beruht auf der Lichtabsorption durch die darüber befindlichen Schichten (Dichte etwa 0,2 pro Emulsionsschicht, gemessen bei rotem Licht), die Verflachung auf dem vertikalen Eberhard-Effekt. Auch die Purpurkurve ist im fertigen Film flacher als im Zweischichtenfilm.

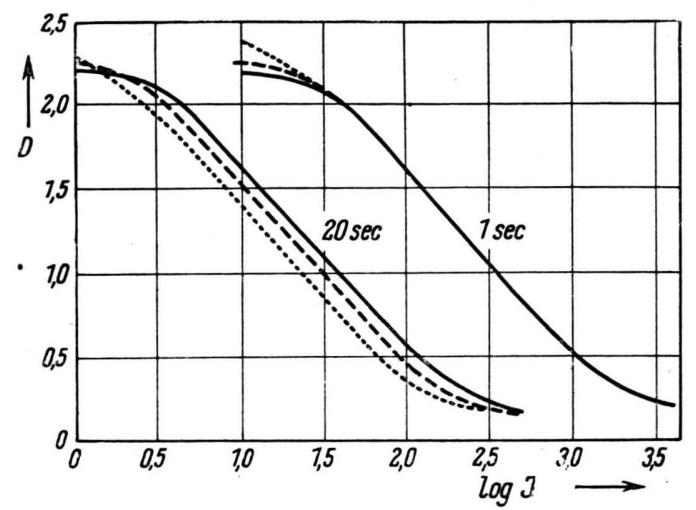

Abb. 3. Schwarzschild-Effekt bei Agfacolor-KunstlichtUmkehrfilm.

Ordinate: Einzelschichtdichten.

Belichtungszeiten 1 sec und 20 sec.

Allgemein kann man sagen, daß die einzeln vergossenen Schichten stets steiler als ein Mehrschichtenguß arbeiten, was bei der Farbfilmfabrikation berücksichtigt werden muß. Durch verlängerte Entwicklung kann man diesen Unterschied mildern.

Der Schwarzschild-Effekt beruht darauf, daß der photochemische Wirkungsgrad von der Belichtungszeit abhängt. Das heißt: Belichtet man eine Schicht mit einer bestimmten Gesamtbelichtung (Lux-Sekunden) einmal kurz und intensiv, das andere mal lange und entsprechend schwächer, so sind die entwickelten Schwärzungen nicht gleich. Im allgemeinen hat der Wirkungsgrad bei einer bestimmten Belichtungszeit ein Maximum und fällt bei sehr langen und sehr kurzen Belichtungszeiten ab. In der SchwarzweißPhotographie spielt der Schwarzschild-Effekt in der Astrophotographie (sehr lange Belichtungszeiten, Herstellung von Astroplatten mit geringem Schwarzschild-
Effekt), in der Tonfilmtechnik (Ultrakurzzeiteffekt, Verflachung der Gradation) und neuerdings bei der Photographie mit Elektronenblitz (ebenfalls Verflachung) eine Rolle. Beim Mehrschichtenfarbfilm treten diese Effekte im Prinzip in derselben Form auf, interessieren hier aber nur insoweit, als sich die einzelnen Schichten verschieden verhalten.

Das ist z. B. beim Agfacolor-Umkehrfilm tatsächlich der Fall, wie aus Abb. 3 hervorgeht. Dort sind die Sensitometerkurven vom Agfacolor-Umkehrfilm eingezeichnet.

Man sieht, daß die bei der Belichtungszeit 1 se zusammenfallenden Gradationskurven bei langen Belichtungszeiten parallel verschoben sind, daß also der ursprünglich neutral grau aussehende Film dann blau aussieht. Man soll es daher möglichst vermeiden, mit sehr langen Belichtungszeiten zu arbeiten, falls man den Farbstich nicht mit einem schwachen Orangefilter kompensieren will.

Bei kürzeren Zeiten tritt der Effekt schwächer auf, so daß der Unterschied zwischen 1 sec und $1 / 20$ see z. B. viel geringer ist. Aus diesem Grunde spielt der Effekt bei Agfacolor-Tageslicht-Umkehrfilm mit den meist kürzeren Belichtungszeiten keine große Rolle.

Auch bei Agfacolor-Negativfilm stört er nicht, da ein Farbstich im Kopierprozeß korrigiert werden kann.

Wichtig ist er noch beim Agfacolor-Diapositivfilm. Wird z. B. dasselbe Negativ in demselben Vergrößerungsapparat unter sonst gleichen Bedingungen einmal im Maßstab 1:3, das andere Mal im Maßstab $1: 10$ auf Positivfilm vergrößert, so sieht das größere Diapositiv, das ja länger belichtet werden mußte, erheblich röter aus (der Farbstich ist naturgemäß entgegengesetzt wie beim Umkehrfilm). Man muß also beim Herstellen der beiden Vergrößerungen stark verschiedene Filter benutzen. Beim Agfacolor-Papier hat man durch geeignete Auswahl der Emulsionen dafür Sorge getragen, daß die einzelnen Schichten gleichen Schwarzschild-Effekt aufweisen, daß also Vergrößerungen in allen Maßstäben mit annähernd gleichem Kopierfilter hergestellt werden können. In der Kinofilmtechnik mit ihren konstanten Belichtungszeiten stört der Schwarzschild-Effekt ebenfalls nicht.

Der Ultrakurzzeit-Effekt wirkt sich in allen Schichten gleich aus. Es genügt also, beispielsweise Agfacolor-Negative, die mit Elektronenblitz belichtet sind, etwas länger als üblich zu entwickeln, um gradationsmäßig ausgeglichene Negative zu erhalten. 